\title{
Two Embedding Theorems with Applications to Weak Convergence and Compactness in Spaces of Additive Type Functions
}

\author{
PASQUALE PORCELLI
}

Communicated by E. HopF

1. Introduction. Let $X$ be a set, $\sigma(X)$ an algebra of subsets of $X$, and $H(X)$ the $\mathrm{NLC}^{1}$ space of bounded additive (not necessarily infinitely additive) functions on $\sigma(X)$, where the norm of the elements $f$ in $H(X)$ is the total variation $V(f, X)$ of $f$ on $\sigma(X)$. The principal object of this paper is to characterize weak convergence of sequences in $H(X)$ and sequential weak compactness of subsets of $H(X)$. We have done this previously (cf. [11], [12]) for the space $B[a, b]$ (the NLC space of all functions that are of ordinary bounded variation on $[a, b]$ and vanish at $a$ ), and one would suspect, since the space $B[a, b]$ is an important example of a space of finitely additive set functions on a set algebra, ${ }^{2}$ that the theorems for $B[a, b]$ should go through to the space $H(X)$. This is so, and, in fact, we establish the theorems for $H(X)$ by embedding separable subspaces of $H(X)$ into $B[0,1]$ in a way that makes it possible to translate the theorems from one space to another, without losing any of their elementary form.

A few preliminary remarks may help crystallize the type of problem we are attacking. Usually, studies of weak convergence and compactness properties of an NLC space, say $S$, is based on a workable knowledge of the adjoint space $S^{*}$. In the case where $S=H(X)$, then, in general, $S^{*}=H(Y)$, where $Y=\sigma(X)$ (cf. [7]). Consequently, since the space $H(Y)$ is usually more complex than $H(X)$, from the point of view of any underlying algebraic or topological structure of the sets $X$ and $Y$, a direct approach may be extremely difficult. Hence what we want is to obtain a weak convergence and compactness theory for $H(X)$ without relying too much on its adjoint space.

The situation in the case of $B[a, b]$ will illustrate our point. First of all, the proofs of the theorems for $B[a, b]$ are sufficiently basic in structure so as to go through verbatim to the case where $\sigma(X)$ is a sigma algebra and we

Sponsored by the U. S. Army under Contract No. Da-11-022-ORD-2059.

1 Our preference for the name NLC space instead of Banach space is explained in [6].

${ }^{2}$ Cf. [8]. 
restrict ourselves to just the infinitely additive elements of $H(X)$. The breakdown of the outright extension of these arguments to the general case is due to the failure of the usual Radon-Nikodym theorem; i.e. we lose a strong hold on the adjoint space. (In the case of $B[a, b]$, we tacitly circumvented this by decomposing separable subspaces of $B[a, b]$ into the direct products of two completely additive spaces.) The failure of this theorem is due, in turn, to the fact that, in general, there exist nondecreasing functions, say $\alpha$, in $H(X)$ and bounded and strictly positive measurable functions, say $f$, with respect to $\sigma(X)$ such that $\alpha(X)=1$ and $\int f d \alpha=0$. This does not imply that weak convergence cannot be characterized by the concept of uniform absolute continuity. Indeed, such a concept does characterize weak convergence; however, we cannot use this concept as our point of departure for a general study of weak convergence in $H(X)$.

The text of the present paper is divided into four sections. In Section 2 we embed $H(X)$ into $B[0,1]$ and present certain supplementary results vital to obtaining the general weak convergence and compactness theorems in Section 3. A second embedding process is carried on in Section 4 which leads to a necessary and sufficient condition for weak convergence in some rather general NLC spaces. This is used to characterize weak convergence in $H(X)$ by uniform absolute continuity. We conclude this paper in Section 5 with some theorems of general type that follow immediately from the work of the preceding section.

Since we make use of the notation and terminology employed in [11] and [12], we have tried to ease in notations and definitions as they are needed and, at the same time, recount some of the pertinent ones from [11] and [12].

2. The First Embedding Theorem. Throughout this paper $X$ shall denote a fixed set and $\sigma(X)$ a fixed algebra of subsets of $X$, i.e. $\sigma(X)$ is a family of subsets of $X$ that contains the null element (hereafter we shall denote the null element by $\theta$ ) and is closed under complementation and finite union; an algebra $\sigma(X)$ is called a sigma algebra if it is closed under countable union. An additive function $\alpha$ on $\sigma(X)$ is a bounded function, ${ }^{3}$ whose domain is $\sigma(X)$ and range is a subset of the real numbers, such that $\alpha(\theta)=0$ and

$$
\alpha(z+Y)+\alpha(z \cdot Y)=\alpha(z)+\alpha(Y)
$$

whenever each of $Y$ and $z$ is in $\sigma(X)$ [we use $E \cdot H$ to denote the intersection of the sets $E$ and $H]$. A partition $P$ of $X$ is a finite collection $\left\{E_{i}\right\}_{i \geqq 1}$ of elements of $\sigma(X)$ such that $E_{i} \cdot E_{i}=\theta, i \neq j$, and $\sum_{i} E_{i}=X$. If $\alpha$ is an additive function on $\sigma(X)$, then $V(\alpha, X)$ denotes the total variation of $\alpha$ and is defined to be the smallest number $M$ such that $\sum_{i}\left|\alpha\left(E_{i}\right)\right| \leqq M$ for all partitions $P$ of $X$. If $E \varepsilon \sigma(X)$, then $V(\alpha, E)$ denotes the total variation of $\alpha$ on $E$ and is defined in a

\footnotetext{
${ }^{3}$ Unbounded functions $\alpha$ such that $|\alpha(E)| \neq \infty, E \varepsilon \sigma(X)$, and $\alpha(Z+Y)+\alpha(Z \cdot Y)=$ $\alpha(Z)+\alpha(Y)$ exist. In fact, this is the same as saying unbounded linear functions exist in any nonzero dimensional NLC space. We include the boundedness as part of our definition for the sake of simplicity.
} 
similar way. We denote the real NLC space of all additive functions on $\sigma(X)$, where the norm is the total variation on $X$, by $H(X)$. If $T$ is a linearly ordered set with a max and a min, say $t$ and $\underline{t}$ (i.e. if $<$ denotes the order relation and $t \varepsilon T$, then $t<t$ if $t \neq t$ and $\underline{t}<t$ if $t \neq t$ ), and $f$ is a function on $T$ into the real numbers, then $f$ is of bounded variation on $T$ if there exists $M \geqq 0$ such that $\sum_{i=0}^{n}\left|f\left(t_{i+1}\right)-f\left(t_{i}\right)\right| \leqq M$ for every subdivision $\underline{t}=t_{0}<\cdots<t_{n+1}=t$ of $T$; the smallest such number $M$ is the total variation of $f$ on $T$ and is denoted by $V(f, T)$. Using the total variation as a norm, the collection of all real-valued functions $f$ that are of bounded variation on $T$ and vanish at $\underline{t}$ is a real NLC space which we denote by $B(T)$.

The principal result of this section is that if $H^{\prime}(X)$ is a separable subspace of $H(X)$, then $H^{\prime}(X)$ can be isomorphically and isometrically embedded into a subspace of $B[0,1]$. We do this by first constructing a set $T$, with the above properties, and then embedding $H^{\prime}(X)$ into a subspace of $B(T)$. Our construction of $T$ gives a simple topology for $T$ and allows us to map it homeomorphically onto a closed subset of $[0,1]$.

Theorem 2.1. If $H^{\prime}(X)$ is a separable subspace of $H(X)$, then there exists a linearly ordered set $T$ such that (1) $T$ has a max and a min, (2) $T$ has the least upper bound property, and (3) there exists a separable subspace $B^{\prime}(T)$ of $B(T)$ such that $H^{\prime}(X)$ and $B^{\prime}(T)$ are isomorphically isometric.

Proof. Let $E_{11}=\theta, E_{21}=X$, and $\left\{h_{i}\right\}_{i=1}^{\infty}$ be a countable dense subset of $H^{\prime}(X)$; for convenience we take $h_{1} \equiv 0$. Let $E_{12}, E_{22}, \cdots, E_{n_{2} 2}$ be a partitioning of $X$ such that (1) $E_{12}=\theta$, (2) $V\left(h_{2} X\right)-\sum_{i=1}^{n_{2}}\left|h_{2}\left(E_{i 2}\right)\right|<1$, and (3) $V\left(h, E_{i 2}\right)-$ $\left|h_{2}\left(E_{i 2}\right)\right|<1$ for $i=1, \cdots, n_{2}$. Proceeding by induction, for each positive integer $s$ let $\left\{E_{i s}\right\}_{i=1}^{n_{s}}$ be a refinement of $\left\{E_{i s-1}\right\}_{\substack{n=1 \\ i=1}}^{n-1}$ such that (1) $E_{1 s}=\theta$, (2) $V\left(h_{m}, X\right)-\sum_{i=1}^{n_{i}}\left|h_{m}\left(E_{i s}\right)\right|<s^{-1}$ for $m=1,2, \cdots, s$, (3) $V\left(h_{m}, E_{i s}\right)-$ $\left|h_{m}\left(E_{i s}\right)\right|<s^{-1}$ for $m=1,2, \cdots, s$ and $i=1,2, \cdots, i_{s}$, and (4) if $u<$ $v \leqq n_{s-1}, E_{i s} \subset E_{u s-1}$, and $E_{i s} \subset E_{v s-1}$, then $i<j$. The last requirement is of vital importance in the construction of $T$. The following figure of the array $\left\{\left\{E_{i s}\right\}_{i=1}^{n_{s}}\right\}_{s=1}^{\infty}$ will help us to describe $T$.

We shall now define $T$ in such a manner that each element of $T$ is a nested sequence of sets taken from the array $\left\{\left\{E_{i s}\right\}_{i=1}^{n_{s}}\right\}_{s=1}^{\infty} ; t \varepsilon T$ if, and only if, there exists a sequence $\left\{H_{p}\right\}_{p=1}^{\infty}$ of elements of $\sigma(X)$ such that (1) $H_{p+1} \subset H_{p}, p=$ $1,2, \cdots$, and (2) for each $p$ there exists an integer $i \leqq n_{p}$ such that $H_{p}=E_{i p}$; we shall call each such sequence $\left\{H_{p}\right\}_{p=1}^{\infty}$ a $T$-chain. If $t \varepsilon T, t^{\prime} \varepsilon T$, and $\left\{H_{p}\right\}_{p=1}^{\infty}$ and $\left\{H_{p}^{\prime}\right\}_{p=1}^{\infty}$ are the $T$-chains that determine $t$ and $t^{\prime}$ respectively, then we say that $t$ precedes $t^{\prime}$ (hereafter, we shall denote this by $t<t^{\prime}$ ) if, and only if, there exist an integer $s$ and integers $i$ and $j$ such that $i<j \leqq n_{s}, H_{p}=E_{i s}$, and $H_{p}^{\prime}=E_{i s}$ for some $p$. In view of (4) of the last paragraph, we see that once the $T$-chains separate, they cannot come back together, i.e. the relation $<$ is a linear ordering of $T$. The elements $\underline{t}$ and $\bar{t}$, defined by the $T$-chains $\left\{E_{1}\right\}_{s=1}^{\infty}$ and $\left\{E_{n s s}\right\}_{s=1}^{\infty}$ respectively, are the minimal and maximal elements of $T$.

Let us suppose now that $T^{\prime}$ is a subset of $T$ and that, for each positive integer $s$, 


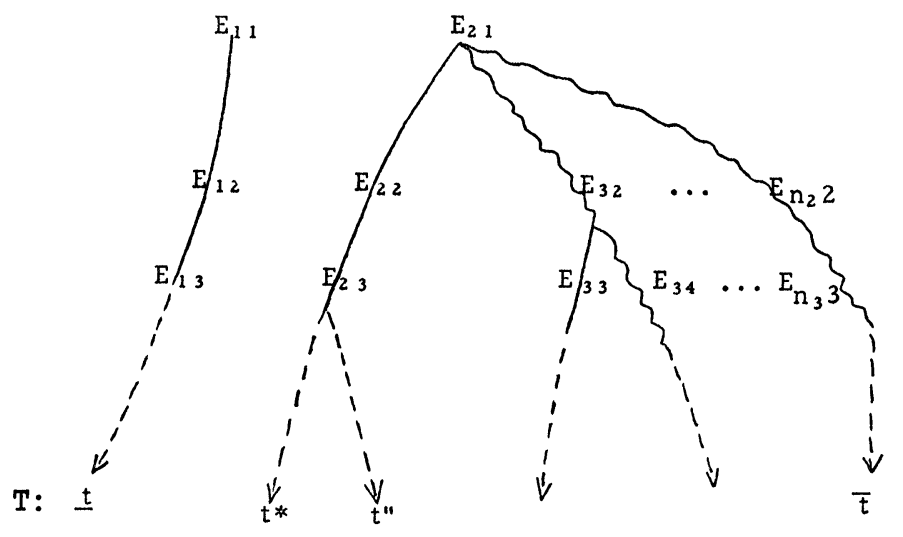

Figure 1.

$i_{s}$ denotes the largest integer less than or equal to $n_{s}$ such that $E_{i s s}$ is the $s^{\text {th }}$ term in a $T$-chain defining some element $t^{\prime}$ of $T^{\prime}$. The element $t$ defined by the $T$-chain $\left\{E_{i_{s s}}\right\}_{s=1}^{\infty}$ is the least upper bound of $T^{\prime}$.

Before going on to the construction of $B^{\prime}(T)$, let us note an important property of $T$. Namely, there exists, at most, a countable collection of pairs $\left(t, t^{*}\right)$ of elements of $T$ such that $t<t^{*}$ and no element of $T$ is between $t$ and $t^{*}$ (e.g. the elements $\underline{t}$ in $t^{*}$ in Figure 1 form such a pair). We shall refer to such a pair as a gap pair. It arises in the following manner: Let $\left\{H_{p}\right\}_{p=1}^{\infty}$ and $\left\{H_{p}^{\prime}\right\}_{p=1}^{\infty}$ be two chains such that (1) there exists $s_{0}$ such that $H_{p}=H_{p}^{\prime}$ for $p \leqq s_{0}$ and (2) if $s>s_{0}$ and $H_{s}=E_{i s}$, then $H_{s}^{\prime}=E_{i+1 s}$; the elements $t$ and $t^{\prime}$ defined by $\left\{H_{p}\right\}_{p=1}^{\infty}$ and $\left\{H_{p}^{\prime}\right\}_{p=1}^{\infty}$ form a gap pair and, except for the pair $\left(\underline{t}, t^{*}\right)$ of Figure 1, all such gap pairs can be described in this manner; also, for descriptive purposes, we say that the pair $\left(t, t^{\prime}\right)$ starts at the $s_{0}+1$ stage of its $T$-chains. In order to see that the collection $S$ of gap pairs is, at most, countable, let us decompose $S$ into $\sum_{n=1}^{\infty} S_{n}$ where, for each $n$, the gap pair $\left(t_{1}, t_{2}\right)$ is in $S_{n}$ if, and only if, $\left(t_{1}, t_{2}\right)$ starts at the $n+1$ stage of its $T$-chains. For each $n, S_{n}$ is a finite set. If we now topologize $T$, using the open segments induced by the linear ordering $<$, we see that the elements belonging to the gap pairs form a dense set. Consequently, under this topology, $T$ is a totally disconnected, separable, and normal Hausdorff space. We shall return to this later.

In order to construct $B^{\prime}(T)$, let $t$ be an element of $T, m$ a positive integer, and, say, $\left\{E_{i_{s} s}\right\}_{s=1}^{\infty}$ the $T$-chain defining $t$. For each positive integer $n$, let $h_{m}^{n}(t)=$ $\sum_{p=1}^{i_{n}} h_{m}\left(E_{p, n}\right)$, so that $h_{m}^{n}(t) \varepsilon B(T)$ and is, in fact, a simple step function on $T$. We shall show now that $\lim _{n} h_{m}^{n}(t)$ exists for $t \varepsilon T$ and defines a function $f_{m}(t) \varepsilon B(T)$. To this end, let us suppose first that $h_{m}$ is nonnegative and nondecreasing on $\sigma(X) ;$ i.e. if $z_{i} \varepsilon \sigma(X), i=1,2$, and $z_{1} \subset z_{2}$, then $0 \leqq h_{m}\left(z_{1}\right) \leqq$ $h_{m}\left(z_{2}\right)$. Under this supposition, we see that, for a fixed $t$, $0 \leqq h_{m}^{n+1}(t) \leqq h_{m}^{n}(t)$, $n=1,2, \cdots$, so that if $f_{m}(t)=\lim _{n} h_{m}^{n}(t)$, then $f_{m} \varepsilon B(T)$. The case where $h_{m}$ is nonnegative and nondecreasing follows from the preceding situation by decom- 
posing $h_{m}$ into the difference of two functions of this type $h_{m}^{\prime}$ and $h_{m}^{\prime \prime}$. This is done in the following way: For each $E \varepsilon \sigma(X)$, set $h_{m}^{\prime}(E)=v\left(h_{m}, E\right)$ and $h_{m}^{\prime \prime}(E)=$ $h_{m}^{\prime \prime}(E)-h_{m}(E)$. The nonnegative and nondecreasing properties of $h_{m}^{\prime}$ and $h_{m}^{\prime r}$ imply that $\lim _{n} h_{m}^{n}(t)$ exists for each $t \varepsilon T$ and defines a function $f_{m} \varepsilon B^{\prime}(T)$. (We note that, in general, the functions $h_{m}^{\prime}$ and $h_{m}^{\prime \prime}$ will not be in $H^{\prime}(X)$.) $B^{\prime}(T)$ denotes the closed linear manifold generated by $\left\{f_{m}\right\}_{m=1}^{\infty}$ in $B(T)$.

It remains to show that $H^{\prime}(X)$ and $B^{\prime}(T)$ are isomorphically isometric. To this end, we shall show that if $\left\{c_{i}\right\}_{i=1}^{n}$ is any set of real numbers, $h=\sum_{i=1}^{n} c_{i} h_{i}$, and $g=\sum_{i=1}^{n} c_{i} f_{i}$, then $V(h, X)=V(g, T)$. Suppose $\epsilon>0$ and $\underline{t}=$ $t_{0}<t_{1}<\cdots<t_{u+1}=t$ is a subdivision of $T$ such that

$$
V(g, T)-\epsilon \leqq \sum_{j=0}^{\mu}\left|g\left(t_{j+1}\right)-g\left(t_{j}\right)\right|=\sum_{j=0}^{\mu}\left|\sum_{i=1}^{n} c_{i}\left\{f_{i}\left(t_{j+1}\right)-f_{i}\left(t_{j}\right)\right\}\right| .
$$

Recalling the definition of $f_{i}$, we see that there exist an integer $s$ and partition $\left\{E_{p}\right\}_{p=1}^{m}$ of $X$ such that (1) for each $p$ there exist integers $v_{p}$ and $w_{p}$ such that $E_{p}=\sum_{1=v_{p}}^{w_{p}} E_{i s}$ and $w_{p}+1=v_{p+1}$ and (2)

$$
\left|\sum_{i=0}^{\mu}\right| \sum_{i=1}^{n} c_{i}\left\{f_{i}\left(t_{i+1}\right)-f_{i}\left(t_{j}\right)\right\}\left|-\sum_{p=1}^{m}\right| \sum_{i=1}^{n} c_{i} h_{i}\left(E_{p}\right)||<\epsilon .
$$

Hence $V(g, T)-\epsilon \leqq V(h, X)+\epsilon$, or $V(g, T) \leqq V(h, X)$. To show that this is actually an equality, let us first note that it implies that $\left\{f_{m}\right\}_{m=1}^{\infty}$ is dense $B^{\prime}(T)$ [replace $g$ by $g-f_{m}$ and $h$ by $h-h_{m}$ ]. Suppose now that $m$ is a positive integer, $\epsilon>0$, and $s$ is a positive integer such that $m<s$ and $s^{-1}<\epsilon$. Hence $V\left(h_{m}, X\right)-\epsilon \leqq \sum_{i=1}^{i_{i}}\left|h_{m}\left(E_{i s}\right)\right|$. Let $\underline{t}=t_{1}<\cdots<t_{i_{s}}=t$ be a subdivision of $T$ such that, for each $i$, the $s^{t h}$ term in the $T$-chain defining $t_{i}$ is $E_{i \text { s }}$. Let $n$ be a positive integer such that $s<n$ and

$$
\sum_{i=2}^{i_{B}}\left|h_{m}^{n}\left(t_{i}\right)-h_{m}^{n}\left(t_{i-1}\right)\right| \leqq \epsilon+\sum_{i=2}^{i_{B}}\left|f_{m}\left(t_{i+1}\right)-f_{m}\left(t_{i-1}\right)\right| \text {. }
$$

There exists a partition $\left\{K_{p}\right\}_{p=1}^{\mu}$ of $X$ such that

$$
\sum_{i=2}^{i_{1}}\left|h_{m}^{n}\left(t_{i}\right)-h_{m}^{n}\left(t_{i-1}\right)\right|=\sum_{p=1}^{\mu}\left|h_{m}\left(K_{p}\right)\right|
$$

and such that $\left\{K_{p}\right\}_{p=1}^{\mu}$ is a refinement of $\left\{E_{i s}\right\}_{i=1}^{i}$. Consequently,

$$
\left|\sum_{i=1}^{i_{s}}\right| h_{m}\left(E_{i s}\right)\left|-\sum_{p=1}^{\mu}\right| h_{m}\left(K_{p}\right)||<2 \epsilon
$$

and upon combining these various inequalities we get

$$
V\left(h_{m}, X\right)-\epsilon \leqq 3 \epsilon+\sum_{i=2}^{i_{9}}\left|f_{m}\left(t_{i+1}\right)-f_{m}\left(t_{i-1}\right)\right|
$$

or $V\left(h_{m}, X\right) \leqq 4 \epsilon+V\left(f_{m}, T\right)$, so that $V\left(h_{m}, X\right) \leqq V\left(f_{m}, T\right)$. Since the previous inequality yields $V\left(f_{m}, T\right) \leqq V\left(h_{m}, X\right)$ [take $g=f_{m}$ and $h=h_{m}$, we have $V\left(f_{m}, T\right)=V\left(h_{m}, X\right)$. Returning now to $V(g, T) \leqq V(h, X)$, let $\epsilon>0$ and 
$m$ be an integer such that $V\left(h_{m}-h, X\right)<\epsilon$. Hence, for this $m$, we have $\left|V(g, T)-V\left(f_{m}, T\right)\right|<\epsilon$ and $\left|V(h, X)-V\left(h_{m}, X\right)\right|<\epsilon$. Now

$$
V(g, T)=\eta+V\left(f_{m}, T\right)=\eta+V\left(h_{m}, X\right)=\eta+\eta^{\prime}+V(h, X),
$$

where $|\eta|<\epsilon$ and $\left|\eta^{\prime}\right|<\epsilon$, so that, finally, $V(h, X)=V(g, T)$. It follows readily now that $H^{\prime}(X)$ and $B^{\prime}(T)$ are isomorphically isometric.

This completes the proof of Theorem 2.1.

Theorem 2.2. The space $B^{\prime}(T)$ in the conclusion of Theorem 2.1 is isomorphically isometric to a subspace $B^{\prime}[0,1]$ of $B[0,1]$.

Proof. Since the number of gap pairs $\left(t, t^{\prime}\right)$ of $T$ is at most countable, we can enumerate all elements $t$ of $T$, which appear as a rightsided term in a gap pair, as a countable sequence, say $\left\{t_{i}\right\}_{i=0}^{\infty}$, where $\underline{t}_{0}=t$. Let $\delta$ be a real-valued function on $T$ such that $\delta(t)=0$ and, for each $t \varepsilon T$ distinct from, $t \delta(t)=\sum_{t_{n} \leq t} 1 / 2^{n}$. If $S$ is in the range of $\delta$ on $[0,1]$, then, since the gap pairs are dense in $T, \delta$ is an order-preserving mapping between $T$ and $S$ and is, in fact, a homeomorphism between $T$ and $S$. We note that while $S$ is a totally disconnected closed subset of $[0,1]$, it is not a Cantor type set (in the sense of a homeomorphism) since it is not a perfect set. However, in general, it contains one or more Cantor type sets as components.

Suppose now $f \varepsilon B(T)$ and, for each $t \varepsilon T, \tau=\delta(t)$. Let $k$ denote the function in $B\left[\begin{array}{ll}0 & 1\end{array}\right]$ such that $k(\tau)=f(t)$ for $t \varepsilon T$ and such that, on the complement $S_{c}$ of $S$ in $[0,1], k$ is linear and continuous on the closure of every open connected set contained in $S_{c}$. This gives a correspondence which is an isomorphism and an isometry between $B(T)$ (and, consequently, $B^{\prime}(T)$ ) and a subspace of $B[0,1]$.

This concludes the proof of Theorem 2.2. As an immediate consequence of this and Theorem 2.1 we have

Corollary 2.2.1. The space $H^{\prime}(X)$ of Theorem 2.1 is isomorphically isometric to a subspace of $B[0,1]$.

As a consequence of Corollary 2.2 .1 and the fact that $B[0,1]$ is weakly complete (cf. [3] and [12]) we have

Corollary 2.2.2. The space $H(X)$ is weakly complete. ${ }^{4}$

We shall now develop the weak convergence and weak compactness conditions for $B(T)$. These conditions follow readily from Theorem 2.2 and the results of [11] and [12]. If $t \varepsilon T$ and $\tau \varepsilon T$ and $t<\tau$, then the subinterval [t, $\tau]$ of $T$ denotes the set of all points $t^{\prime} \varepsilon T$ such that $t \leqq t^{\prime} \leqq \tau$. Finally, let us define $\Gamma(T)$ as the collection of all systems $\left\{\left[t_{p}, \tau_{p}\right]\right\}_{p \gtrless 1}$, infinite or finite, of nonoverlapping subintervals of $T$.

Theorem 2.3. If $f_{n} \varepsilon B(T), n=1,2, \cdots$, then $\left\{f_{n}\right\}_{n=1}^{\infty}$ is a weakly convergent

\footnotetext{
4 This was shown by S. KaKUTANI (Th. 12, [10]).
} 
sequence if, and only if,

$$
\lim _{n} \sum_{p \geq 1}\left[f_{n}\left(\tau_{p}\right)-f_{n}\left(t_{p}\right)\right]
$$

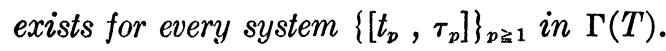

This is the equivalent of Theorem 3.1 of [12]. We note that $\left\{f_{n}\right\}_{n=1}^{\infty}$ will converge weakly to zero when, and only when, the limit in (2.3a) is zero for every system in $\Gamma(T)$ (cf. [12], Cor. 2.1.1). Also, in this case, the expressions $\left[f\left(\tau_{p}\right)-f\left(t_{p}\right)\right]$ in (2.3a) can be replaced by $\left|f\left(\tau_{p}\right)-f\left(t_{p}\right)\right|(c f$. [12], Th. 2.1 or [9], Cor. 2). This special case will be of some importance to us later, so we shall state it as

Corollary 2.3.1. If $f_{n}$ \& $B(T)$, then $\left\{f_{n}\right\}_{n=1}^{\infty}$ converges weakly to zero if, and only if,

$$
\lim _{n} \sum_{p}\left[f_{n}\left(\tau_{p}\right)-f_{n}\left(t_{p}\right)\right]=0
$$

or, equivalently,

$$
\lim _{n} \sum_{p}\left|f_{n}\left(\tau_{p}\right)-f_{n}\left(t_{p}\right)\right|=0
$$

for every system $\left\{\left[t_{p}, \tau_{p}\right]\right\}_{p \geqq 1}$ in $\Gamma(T)$.

A subset $B_{0}$ of $B(T)$ is said to be uniformly bounded in case there exists $M \geqq 0$ such that $V(f, T) \leqq M$ for every $f$ in $B_{0}$. If a sequence $\left\{f_{n}\right\}_{n=1}^{\infty}$ in $B_{0}$ is such that for each $\epsilon>0$ and each system $\left\{\left[t_{p}, \tau_{p}\right]\right\}_{p \geq 1} \varepsilon \Gamma(T)$ there exists $p_{\mathrm{c}} \geqq 0$ such that

$$
\sum_{p \geq p_{0}}\left|f_{n}\left(\tau_{p}\right)-f_{n}\left(t_{p}\right)\right|<\epsilon
$$

for $n=1,2, \cdots$, then $\left\{f_{n}\right\}_{n=1}^{\infty}$ is said to satisfy (2.4a). $B_{0}$ is said to satisfy (2.4a) if each sequence in $B_{0}$ satisfies (2.4a). Corresponding to Theorem 4.1 of [12], we have

Theorem 2.4. $A$ subset $B_{0}$ of $B(T)$ is sequentially weakly compact if, and only if, $B_{0}$ is uniformly bounded and $B_{0}$ satisfies (2.4a).

As we remarked in [12], the uniform boundedness requirement can be considerably weakened and is in some instances redundant (cf. [12], Th. 4.2).

As an analogue to Theorem 4.3 of [12], we have

Theorem 2.5. $A$ subset $B_{0}$ of $B(T)$ is sequentially weakly compact if, and only if, every sequence, say $\left\{f_{n}\right\}_{n=1}^{\infty}$, in $B_{0}$ contains a subsequence, say $\left\{f_{n}\right\}_{i=1}^{\infty}$, such that

$$
\lim _{i} \sum_{p}\left|f_{n_{i}}\left(\tau_{p}\right)-f_{n_{i}}\left(t_{p}\right)\right|
$$

exists for every system $\left\{\left[t_{p}, \tau_{D}\right\}_{p_{\geq \geq 1}}\right.$ in $\Gamma(T)$.

5 This is the same as $\lim _{m, n} \sum_{p=n}^{\infty}\left|f_{m}\left(\tau_{p}\right)-f_{m}\left(t_{p}\right)\right|=0$. Starting in Section 3, we shall use this formulation of the condition. 
We shall conclude this section with a result concerning sequences in $B(T)$ which are not weakly convergent but satisfy certain pointwise and local conditions. This result will be of considerable importance in the next section in that it will enable us to carry over Theorems $2.3-2.5$, in a sensible way, to theorems about $H(X)$. The notation we use here is self-evident, however; for a formulation of it see Section 3 of [11].

Theorem 2.6. Suppose $f_{n} \varepsilon B(T), n=1,2, \cdots,\left\{f_{n}\right\}_{n=1}^{\infty}$ does not converge weakly, $\lim _{n} f_{n}(t)=0$ for each $t \varepsilon T$, and for $\epsilon>0$ and $t_{c} \varepsilon T$ there exists a segment $\lambda$ containing $t_{0}$ such that $V\left(f_{n}, \lambda\right)<\epsilon$ for $n=1,2, \cdots$. Then there exist $\delta>0$, a system $\left\{\left[t_{p}, \tau_{p}\right]\right\}_{p \geqq 1} \varepsilon \Gamma(T)$, and a subsequence $\left\{f_{n_{i}}\right\}_{i=1}^{\infty}$ of $\left\{f_{n}\right\}_{n=1}^{\infty}$ such that (1)

$$
\liminf _{i} \sum_{p \geqq 1}\left|f_{m_{i}}\left(\tau_{p}\right)-f_{m_{i}}\left(t_{p}\right)\right| \geqq \delta
$$

and (2) for each $p t_{p}$ (and $\tau_{p}$ ) is not a limit point of the set $\left[x ; x=t_{i}, x=\tau_{i}\right.$, $i=1,2, \cdots]$.

Proof. In view of Theorem 2.2, we can take $T=[0,1]$. Since $\left\{f_{n}\right\}_{n=1}^{\infty}$ does not converge weakly, it obviously does not converge weakly to zero, that so from Corollary 2.3.1 (in particular, (2.3c)) there exists $\delta_{1}>0$, a subsequence $\left\{f_{m_{n}}\right\}_{n=1}^{\infty}$ of $\left\{f_{n}\right\}_{n=1}^{\infty}$, and, in view of the point-wise convergence of $\left\{f_{n}\right\}_{n=1}^{\infty}$, an infinite system $\left\{\left[a_{p}, b_{p}\right]\right\}_{p=1}^{\infty} \varepsilon \Gamma([a, b])$ such that

$$
\lim _{n} \inf \sum_{p=1}^{\infty}\left|f_{m_{n}}\left(b_{p}\right)-f_{m_{n}}\left(a_{p}\right)\right|>3 \delta_{1} \text {. }
$$

For convenience we take $m_{n}=n, n=1,2, \cdots$.

Starting with $f_{1}$, there exists $p_{1}>0$ such that $\sum_{p \geqq p_{1}}\left|f_{1}\left(b_{p}\right)-f_{1}\left(a_{p}\right)\right|<\frac{1}{2} \delta_{1}$ and there exists a least integer $m_{2}$ such that $\sum_{p=1}^{p_{1}}\left|f_{m}\left(b_{p}\right)-f_{m}\left(a_{p}\right)\right|<\frac{1}{2} \delta_{1}$ for $m \geqq m_{2}$. There exist mutually exclusive segments $\lambda_{1}, \lambda_{2}, \cdots, \lambda_{p_{1}}$ and $\beta_{1}, \beta_{2}, \cdots, \beta_{p_{1}}$ containing $a_{1}, a_{2}, \cdots, a_{p_{1}}$ and $b_{1}, b_{2}, \cdots, b_{p_{1}}$ respectively such that $\sum_{i=1}^{p_{1}}\left[V\left(f_{m}, \lambda_{i}\right)+V\left(f_{m}, \beta_{i}\right)\right]<\frac{1}{2} \delta_{1}$. Let us delete from $\left.\left\{a_{p}, b_{p}\right]\right\}_{p=p_{1}+1}^{\infty}$ all intervals which are contained in some $\lambda_{i}$ or $\beta_{i}, i=1,2, \cdots, p_{1}$. (We lose no generality in assuming that $\left[a_{p}, b_{p}\right], p>p_{1}$, is either contained in some $\lambda_{i}$ or $\beta_{i}$ or intersects no $\lambda_{i}$ or $\beta_{i}$.) Since this deletion effects the value of each $f_{m}$ by at most $\frac{1}{2} \delta_{1}$, infinitely many intervals are left. The direction of the proof is now apparent; starting with $f_{m}$, and the remaining intervals of $\left\{\left[a_{p}, b_{p}\right]\right\}_{p=p_{1}+1}^{\infty}$ we repeat the above argument replacing $\frac{1}{2} \delta_{1}$ by $\frac{1}{4} \delta_{1}$. Hence, proceeding in this way, we obtain a subsequence $\left\{f_{m i}\right\}_{i=1}^{\infty}, m_{1}=1$, of $\left\{f_{m}\right\}_{m=1}^{\infty}$ and a subsequence $\left\{\left[t_{p}, \tau_{p}\right]\right\}_{p=1}^{\infty}$ of $\left\{\left[a_{p}, b_{p}\right]\right\}_{p=1}^{\infty}$ such that $\sum_{p}\left|f_{m_{i}}\left(t_{p}\right)-f_{m_{i}}\left(\tau_{p}\right)\right| \geqq \delta_{1}$ for $i=1,2, \cdots$. Finally, it is clear from our proof that the conclusion of Theorem 2.6 can be changed so as to read

$$
\lim \inf _{i}\left|\sum_{p}\left[f_{m_{i}}\left(t_{p}\right)-f_{m_{i}}\left(\tau_{p}\right)\right]\right| \geqq \delta .
$$

3. Weak Convergence and Compactness in $H(X)$. In this section we shall 
derive the principal theorems characterizing weak convergence and weak compactness in the space $H(X)$. In addition to our previous notation we let $\Gamma(\sigma(X))$ denote the collection of all systems $\left\{E_{p}\right\}_{p \geqq 1}$, finite or infinite, of nonoverlapping elements of $\sigma(X)$.

Throughout this section we shall tacitly assume the results of Section 2; e.g. if $\alpha_{m} \varepsilon H(X), m=1,2, \cdots$, and if we say $f_{m}$ is the image of $\alpha_{m}$ in $B(T)$, then we imply that, starting with the closed linear manifold $H^{\prime}(X)$ generated by $\left\{\alpha_{m}\right\}_{m \geqslant 1}$ in $H(X)$, we have gone through the process used in the proof of Theorem 2.1, constructed $T$ and $B^{\prime}(T)$, and found that $f_{m}$ is the image $\alpha_{m}$ under the isomorphic and isometric mapping between $H^{\prime}(X)$ and $B^{\prime}(T)$. Also, in this direction, we denote by $F$ the set of all elements $E_{i s}, i=1,2, \cdots, n_{s}$, $i=1,2, \cdots$, that appeared in the proof of Theorem 2.1. $\Gamma(F)$ denotes the collection of all systems $\left\{E_{p}\right\}_{p_{\grave{1} 1}} \varepsilon \Gamma(\sigma(X))$ such that, for each $p$, there exists an integer $s$ so that $E_{p}=\sum_{i} E_{j s}$, where $j$ sums over a subset of $1,2, \cdots, n_{s}$.

We shall need the following lemmas.

Lemma 3.1. If $\alpha_{m} \varepsilon H(X), m=1,2, \cdots, \lim _{m} \sum_{p \geqq 1} \alpha_{m}\left(H_{p}\right)=0$ for each system $\left\{H_{p}\right\}_{p \geq 1}$ in $\Gamma(\sigma(X))$, and $\left\{E_{i}\right\}_{i=1}^{\infty}$ is a sequence of subsets of $\sigma(X)$ such that $E_{i+1} \subset E_{i}\left(\right.$ or $\left.E_{i} \subset E_{i+1}\right), i=1,2, \cdots$, then $\lim _{m} \lim _{n} \alpha_{m}\left(E_{n}\right)=0$.

Proof. We first note that the hypothesis of the lemma implies that $\lim _{m} \alpha_{m}(E)=0$ for every $E \varepsilon \sigma(X)$. Since $\left\{E_{i}-E_{i+1}\right\}_{i=1}^{\infty}$ is in $\Gamma(\sigma(X))$, we have $\sum_{i=1}^{\infty} \alpha_{m}\left(E_{i}-E_{i+1}\right) \rightarrow 0$ as $m \rightarrow \infty$. Hence, for $\epsilon>0$, there exists $m_{0}>0$ such that $\left|\sum_{i=1}^{\infty} \alpha_{m}\left(E_{i}-E_{i+1}\right)\right|<\frac{1}{3} \epsilon$ and $\left|\alpha_{m}\left(E_{1}\right)\right|<\frac{1}{3} \epsilon$ provided $m>m_{0}$. Consequently, since

and

$$
E_{n+1}=E_{1}-\sum_{p=1}^{n}\left(E_{p}-E_{p+1}\right)
$$

$$
\alpha_{m}\left(E_{n+1}\right)=\alpha_{m}\left(E_{1}\right)-\sum_{p=1}^{n} \alpha_{m}\left(E_{0}-E_{p+1}\right)
$$

for each $m>m_{0}$ there exists $n_{0}$ such that $\left|\alpha_{m}\left(E_{n+1}\right)\right|<\epsilon$ provided $n>n_{0}$ (i.e. for fixed $\left.m, \sum_{p \geqq n_{0}}\left|\alpha_{m}\left(E_{p}-E_{p+1}\right)\right|<\frac{1}{3} \epsilon\right)$. Now this implies that

$$
\lim _{m}\left(\lim _{n} \inf \left|\alpha_{m}\left(E_{n}\right)\right|\right)=0 \text {, }
$$

but, since $\alpha_{m}$ is additive and $E_{i+1} \subset E_{i}$ so that $\lim _{n} \alpha_{m}\left(E_{n}\right)$ exists for each $m$, this, in turn, implies that $\lim _{m} \lim _{n}\left|\alpha_{m}\left(E_{n}\right)\right|=0$. The proof of the case where $E_{i} \subset E_{i+1}, i=1,2, \cdots$, proceeds in the same manner; here, however, we consider the system $\left\{\left(E_{i+1}-E_{i}\right)\right\}_{i=0}^{\infty}, E_{0}=\theta$, and write

$$
E_{n+1}=E_{1}+\sum_{p=1}^{n}\left(E_{p+1}-E_{1}\right) \text {. }
$$

Finally, we note that in the hypothesis of Lemma 3.1 we can replace the condition $\lim _{m} \sum_{p \geq 1} \alpha_{m}\left(H_{p}\right)=0$ with the equivalent condition $\lim _{m} \sum_{p \geq 1}\left|\alpha_{m}\left(H_{p}\right)\right|=0$.

This completes the proof of Lemma 3.1. 
Lemma 3.2. Suppose $\alpha_{m} \varepsilon H(X), m=1,2, \cdots, \lim _{m} \sum_{p \geqq 1} \alpha_{m}\left(E_{p}\right)=0$ for each system $\left\{E_{p}\right\}_{p \geqq 1} \varepsilon \Gamma(F)$, and $f_{m}, m=1,2, \cdots$, is the image of $\alpha_{m}, m=$ $1,2, \cdots$, in $B(T)$. Then, for each $t \in T$,

$$
\lim _{m} f_{m}(t)=\lim _{m} f_{m}(t+)=\lim _{m} f_{m}(t-)=0 .
$$

Proof. Since all these limits arise in the same manner, we shall just establish that $f_{m}(t+) \rightarrow 0$ as $m \rightarrow \infty$. This follows readily from Lemma 3.1 and the fact that, for each $t \varepsilon T$, there exists a nested sequence $\left\{K_{i}\right\}_{i>1}$ of sums of elements of $F$ such that $\lim _{i} \alpha_{m}\left(K_{i}\right)=f_{m}(t+)$.

Lemma 3.3. Under the hypothesis of Lemma 3.2, we have that, for each $\epsilon>0$ and each $t \varepsilon T$, there exists a segment $\lambda$ containing $t$ such that $V\left(f_{m}, \lambda\right)<\epsilon$ for $m=1,2, \cdots$.

Proof. Suppose $t_{0} \varepsilon T$ and $\epsilon>0$ such that if $\lambda$ is a segment containing $t_{0}$. Then there exists a positive integer $m$ such that $V\left(f_{m}, \lambda\right)>20 \epsilon$. In view of the fact that

$$
\lim _{m} f_{m}\left(t_{0}\right)=\lim _{m} f_{m}\left(t_{0}-\right)=\lim _{m} f_{m}\left(t_{0}+\right)=0,
$$

we can find (as in the proof of Lemma 3.5 of [11]) a system $\left\{\left[t_{p}, \tau_{p}\right]\right\}_{p=1}^{\infty} \varepsilon \Gamma(T)$ such that, say, $\tau_{p}<t_{p+1} \rightarrow t_{0}$ as $p \rightarrow \infty$ and a subsequence $\left\{f_{m_{n}}\right\}_{n=1}^{\infty}$ of $\left\{f_{m}\right\}_{m=1}^{\infty}$ such that

$$
\left|\sum_{p=1}^{\infty}\left[f_{m_{n}}\left(\tau_{p}\right)-f_{m_{n}}\left(t_{p}\right)\right]\right| \geqq 10 \epsilon
$$

for $n=1,2, \cdots$. For convenience we take $m_{n}=n$.

Starting now with $f_{1}$ and $\alpha_{1}$, there exists an integer $p_{1}$ and a finite number, say $H_{i}, i=1, \cdots, n_{1}$, of mutually exclusive sets of sums of elements in $F$ such that (1) $\sum_{p>p_{1}} V\left(f_{1},\left[t_{p}, \tau_{p}\right]\right)<\frac{1}{4} \epsilon,(2)$

$$
|| \sum_{i=1}^{n_{1}} \alpha_{1}\left(H_{i}\right)|-| \sum_{p=1}^{p_{1}}\left[f_{1}\left(\tau_{p}\right)-f_{1}\left(t_{p}\right)\right] \mid<\frac{1}{4} \epsilon,
$$

and (3) due to the fact that $t_{p}<\tau_{p+1}<t_{p+1}, p=1,2, \cdots$, for each $p>p_{1}$ and each $i \leqq n_{1}, H_{i}$ is not an element of the $T$-chain defining $t_{p}$ or $\tau_{p}$. Due to the pointwise convergence of $\left\{\alpha_{m}\right\}_{m=1}^{\infty}$ on $F$ and $\left\{f_{m}\right\}_{m=1}^{\infty}$ on $T$, there exists a smallest integer, say $m_{2}$, such that $\sum_{i=1}^{n_{1}}\left|\alpha_{m}\left(h_{i}\right)\right|<\frac{1}{4} \epsilon$ and

$$
\sum_{p=1}^{p_{1}}\left|f_{m}\left(\tau_{p}\right)-f_{m}\left(t_{p}\right)\right|<\frac{1}{4} \epsilon
$$

for $m \geqq m_{2}$.

Going now to $f_{m}$, and $\alpha_{m}$, there exists an integer $p_{2}$ and a finite number, say $H_{i}, i=n_{1}+1, \cdots, n_{2}$, of sums of sets in $F$ such that (1) $H_{i} \cdot H_{i}=\theta$, $i \neq j, i, j=1,2, \cdots, n_{2},(2) \sum_{p>p}, V\left(f_{m},\left[t_{p}, \tau_{p}\right]\right)<\epsilon / 4^{2},(3)$ 


$$
\left\|\sum_{i=n_{1}+1}^{n_{2}} \alpha_{m_{2}}\left(H_{i}\right)|-| \sum_{p=p_{1}+1}^{p_{2}}\left[f_{m_{2}}\left(\tau_{p}\right)-f_{m}\left(t_{p}\right)\right]\right\|<\epsilon / 4^{2},
$$

and (4) for each $p>p_{2}$ and each $i \leqq n_{2}, H_{i}$ is not an element of the $T$-chain defining $t_{p}$ or $\tau_{p}$.

It is clear now that by following the procedure of the last two paragraphs, we obtain a subsequence $\left\{\alpha_{m_{i}}\right\}_{i=1}^{\infty}, m_{1}=1$, of $\left\{\alpha_{m}\right\}_{m=1}^{\infty}$ and a system $\left\{H_{p}\right\}_{p=1}^{\infty} \varepsilon \Gamma\left(F^{\prime}\right)$ such that $\left|\sum_{p=1}^{\infty} \alpha_{m_{i}}\left(H_{p}\right)\right|>8 \epsilon, i=1,2, \cdots$. This contradiction establishes Lemma 3.2.

Lemma 3.4. Suppose $\alpha_{m} \varepsilon H(X), m=1,2, \cdots$, and $\lim _{m} \sum_{p \geqq 1} \alpha_{m}\left(H_{p}\right)$ exists for every system $\left\{H_{p}\right\}_{p \geqq 1}^{\infty} \varepsilon \Gamma(F)$. Then there exists $p_{1}>0$ such that $\sum_{p \geqq p}\left|\alpha_{m}\left(E_{p}\right)\right|<\epsilon$.

This lemma is the analogue of Theorem 2.3 of [11] and the proof follows readily from Corollary 1 of [9].

Theorem 3.1. If $\alpha_{m} \varepsilon H(X), m=1,2, \cdots$, then $\left\{\alpha_{m}\right\}_{m=1}^{\infty}$ is weakly convergent to zero if, and only if,

$$
\lim _{m} \sum_{p \geqq 1} \alpha_{m}\left(E_{p}\right)=0
$$

for every system $\left\{E_{p}\right\}_{p \geqq 1} \varepsilon \Gamma(\sigma(X))$.

Proof. Necessity. If $\left\{E_{p}\right\}_{p \geqq 1} \varepsilon \Gamma(\sigma(X))$, define $L(\alpha)=\sum_{p \geqq 1} \alpha\left(E_{p}\right)$ for $\alpha \varepsilon H(X)$. Since $L \varepsilon H^{*}(X)$, the necessity follows readily from the definition of weak convergence.

Sufficiency. Suppose the contrary, so that if $\left\{f_{m}\right\}_{m=1}^{\infty}$ is the image sequence of $\left\{\alpha_{m}\right\}_{m=1}^{\infty}$ in $B^{\prime}(T)$, then $\left\{f_{m}\right\}_{m=1}^{\infty}$ does not converge weakly to zero. In accordance with Corollary 2.3.1, there exists $\epsilon>0$, a system $\left\{\left[t_{p}, \tau_{p}\right]\right\}_{p \geqq 1} \varepsilon \Gamma(T)$, and a subsequence $\left\{f_{m i}\right\}_{i=1}^{\infty}$ of $\left\{f_{m}\right\}_{m=1}^{\infty}$ such that

$$
\left|\sum_{p \geqq 1}\left[f_{m_{i}}\left(\tau_{p}\right)-f_{m_{i}}\left(t_{p}\right)\right]\right| \geqq 10 \epsilon
$$

$i=1,2, \ldots$. For convenience we drop the absolute value bars on this inequality and also take $m_{i}=i$. In view of Lemmas 3.1 and 3.2, we can invoke Theorem 2.6 and require that no $t_{p}$ or $\tau_{p}$ be a limit point of the set $\left[x ; x=t_{p}\right.$ or $x=\tau_{p}, p=$ $1,2, \cdots]$. Because of this we can proceed exactly as in the proof of Lemma 3.3 (this closure property of $t_{p}$ and $\tau_{p}$ takes the place of the monotonicity properties of $t_{p}$ and $\tau_{p}$ used in that proof) and obtain a subsequence $\left\{\alpha_{m_{i}}\right\}_{i=1}^{\infty}$ of $\left\{\alpha_{m}\right\}_{m=1}^{\infty}$ and a system $\left\{H_{p}\right\}_{p=1}^{\infty} \varepsilon \Gamma\left(F^{\prime}\right)$ such that $\left|\sum_{p=1}^{\infty} \alpha_{m i}\left(H_{p}\right)\right|>8 \epsilon, i=1,2, \cdots$. Since $\Gamma(F) \subset \Gamma(\sigma(X))$, this contradicts (3.1a) and thus completes the proof of Theorem 3.1.

Corollary 3.1.1. If $\alpha_{m} \varepsilon H(X)$, then $\left\{\alpha_{m}\right\}_{m=1}^{\infty}$ is wealkly convergent if, and only if,

$$
\lim _{m} \sum_{p \geqq 1} \alpha_{m}\left(E_{p}\right)
$$

exists for every system $\left\{E_{p}\right\}_{p \geqq 1} \varepsilon \Gamma(\sigma(X))$. 
Proof. The necessity follows by the argument used in Theorem 3.1. (Also, it is interesting, and perhaps useful, to note that it can be obtained from Corollary 2.2.2 and Theorem 3.1.) For the sufficiency, let us, since (3.1b) implies pointwise convergence, set $\alpha_{0}(E)=\lim _{m} \alpha_{m}(E)$ for every $E \varepsilon \sigma(X)$. From this we have

$$
\alpha_{0}\left(E+E^{\prime}\right)+\alpha_{0}\left(E \cdot E^{\prime}\right)=\alpha_{0}(E)+\alpha\left(E^{\prime}\right),
$$

$E, E^{\prime}$ in $\sigma(X)$, so that $\alpha_{0}$ will be in $H(X)$ if we show it is bounded. To this end, suppose the sequence $\left\{V\left(\alpha_{m}, X\right)\right\}_{m=1}^{\infty}$ is unbounded, so that there exist increasing sequences $\left\{n_{p}\right\}_{p=1}^{\infty}$ and $\left\{m_{p}\right\}_{p=1}^{\infty}$ of positive integers such that $n_{p}<m_{p}<n_{p+1}$ and $V\left(\alpha_{n_{p}}-\alpha_{m_{p}}, X\right) \rightarrow \infty$ as $p \rightarrow \infty$. However, the sequence $\left\{\alpha_{n_{p}}-\alpha_{m_{p}}\right\}_{p=1}^{\infty}$ satisfies (3.1a) so that, by Theorem 3.1, $\left\{\alpha_{n_{p}}-\alpha_{m_{p}}\right\}_{p=1}^{\infty}$ is weakly convergent to zero. Consequently $\left\{V\left(\alpha_{m}, X\right)\right\}_{m=1}^{\infty}$ is uniformly bounded and hence $\alpha_{0}$ is in $H(X)$. It now follows, from the definition of $\alpha_{0}$ and Lemma 3.4, that $\left\{\alpha_{m}-\alpha_{0}\right\}_{m=1}^{\infty}$ satisfies (3.1a). Hence $\left\{\alpha_{m}\right\}_{m=1}^{\infty}$ is weakly convergent.

Lemma 3.5. If in the hypothesis of Lemmas 3.1-3.3 we replace the condition $\lim _{m} \sum_{p} \alpha_{m}\left(E_{p}\right)=0$ (relative to $\Gamma(F)$ or $\Gamma(\sigma(X)$ ) with the conditions that $\lim _{m} \alpha_{m}(E)=0$ for every $E \& F$ and

$$
\limsup _{n}\left(\lim _{m} \sum_{p \geqq n}\left|\alpha_{m}\left(E_{p}\right)\right|\right)=0
$$

for every system $\left\{E_{p}\right\}_{p \geqq 1} \varepsilon \Gamma(\sigma(X))$, then the conclusions of Lemmas 3.2 and 3.3 remain valid and the conclusion of Lemma 3.1 remains valid provided the sets $E_{i}$, $i=1,2, \cdots$, in the hypothesis of Lemma 3.1 are finite sums of pairwise nonintersecting elements of $F$.

We shall not prove this lemma since it would amount to a reiteration of the previous arguments. We note, however, in regard to the reformulation of Lemma 3.1, that if $E \subset E^{\prime} \varepsilon F$, then

$$
\alpha_{m}\left(E^{\prime}-E\right)=\alpha_{m}\left(E^{\prime}\right)-\alpha_{m}(E) \rightarrow 0
$$

as $m \rightarrow \infty$ even though $E^{\prime}-E$ may not belong to $F$. The role of Lemma 3.5 is to allow us to invoke Theorem 2.6 in reducing the next theorem to Theorem 2.4.

Theorem 3.2. A subset $H_{0}$ of $H(X)$ is weakly sequentially compact if, and only if, $H_{0}$ is uniformly bounded (i.e. there exists $M \geqq 0$ such that $V(\alpha, X) \leqq M$ for every $\alpha$ in $H_{0}$ ) and for each sequence $\left\{\alpha_{m}\right\}_{m=1}^{\infty}$ in $H_{0}$

$$
\lim _{m, n} \sum_{p \geqq n}\left|\alpha_{m}\left(E_{p}\right)\right|=0
$$

for every system $\left\{E_{p}\right\}_{p \gtrless 1} \varepsilon \Gamma(\sigma(X))$.

Proof. The necessity can be established by exactly the argument used in the proof of Theorem 4.1 of [12] and we shall not repeat it here. Suppose now $\alpha_{m} \varepsilon H_{0}, m=1,2, \cdots . H^{\prime}(X)$ is the closed linear manifold generated by $\left\{\alpha_{m}\right\}_{m=1}^{\infty}$ in $H(X)$. Letting $T, B^{\prime}(T)$, and $F$ have their usual meanings, we select (using 
the uniform boundedness of $H_{0}$, the fact that $F$ is countable, and the diagonalization process) a subsequence $\left\{\alpha_{m_{i}}\right\}_{i=1}^{\infty}$ of $\left\{\alpha_{m}\right\}_{m=1}^{\infty}$ such that $\lim _{i} \alpha_{m_{i}}(E)$ exists for each $E \varepsilon F$. If $\left\{\alpha_{m_{i}}\right\}_{i=1}^{\infty}$ is not weakly convergent, then there exists a sequence $\left\{\beta_{m}\right\}_{m=1}^{\infty}$ such that (1) for each $m$ there exists $m_{i}$ and $m_{i}$ such that $i<j$ and $\beta_{m}=\alpha_{m_{i}}-\alpha_{m_{i}}$, (2) if $m<n$, then $m_{i}<n_{i}<m_{i+1}$, (3) $\left\{\beta_{m}\right\}_{m=1}^{\infty}$ satisfies (3.1c), (4) $\beta_{m}(E) \rightarrow 0$ as $m \rightarrow \infty$ for $E \varepsilon F^{\prime}$, and (5) $\left\{\beta_{m}\right\}_{m=1}^{\infty}$ is not weakly convergent to zero. We note, also, that $\beta_{m} \varepsilon H^{\prime}(X){ }^{6}$ If $f_{m}$ is the image of $\beta_{m}$ in $B^{\prime}(T)$, $m=1,2, \cdots$, then, in view of Lemma 3.5, $\left\{f_{m}\right\}_{m=1}^{\infty}$ satisfies the hypothesis of Theorem 2.6. Because of this, the argument used in the proof of the sufficiency part of Theorem 3.1 establishes Theorem 2.4 for $\left\{f_{m}\right\}_{m=1}^{\infty}$. Hence every subsequence of $\left\{f_{m}\right\}_{m=1}^{\infty}$ contains in turn a weakly convergent subsequence and since $f_{m}(t) \rightarrow 0$ as $m \rightarrow \infty$, it follows that $\left\{f_{m}\right\}_{m=1}^{\infty}$ is weakly convergent to zero. Since $H^{\prime}(X)$ and $B^{\prime}(T)$ are isomorphically isometric, $\left\{\beta_{m}\right\}_{m=1}^{\infty}$ converges weakly to zero. This contradicts (5) above and completes the proof of Theorem 3.2.

Theorem 3.3. A subset $H_{0}$ of $H(X)$ is weakly sequentially compact if, and only if, each sequence $\left\{\alpha_{m}\right\}_{m=1}^{\infty}$ in $H_{0}$ contains a subsequence $\left\{\alpha_{m_{i}}\right\}_{i=1}^{\infty}$ such that

$$
\lim _{i} \sum_{p \geqq 1}\left|\alpha_{m i}\left(E_{p}\right)\right|
$$

for every system $\left\{E_{p}\right\}_{p \geqq 1}$ in $\Gamma(\sigma(X))$.

Proof. The necessity follows from the definition of weak sequential compactness, Corollary 3.1.1, and the fact that a sequence $\left\{\alpha_{m_{i}}\right\}_{i=1}^{\infty}$ satisfying (3.1b) must also satisfy (3.1d) (cf. Corollary 3 of [9] or Theorem 2.2 of [12]). The type of argument used throughout this section shows that if $f_{m}$ is the image of $\alpha_{m}, m=1,2, \cdots$, in $B^{\prime}(T)$, then $\left\{f_{m}\right\}_{m=1}^{\infty}$ satisfies (2.5a) and hence, by Theorem 2.5, $\left\{f_{m}\right\}_{m=1}^{\infty}$ contains a weakly convergent subsequence. Consequently $H_{0}$ is sequentially weakly compact.

This completes the proof of Theorem 3.3.

We shall now turn to the case where $\sigma(X)$ is a sigma algebra and $\alpha_{m}$ is infinitely additive. O. NIKodym (cf. [10']) showed that, in this setting, if $\lim _{m} \alpha_{m}(E)$ exists for every $E \varepsilon \sigma(X)$, then $\lim _{m, n} \sum_{n}^{\infty}\left|\alpha_{m}\left(E_{n}\right)\right|=0$ for every system in $\Gamma(\sigma(X))$. Hence the following is NIKodym's theorem stated in terms of weak convergence:

Theorem 3.4. If $\sigma(X)$ is a sigma algebra and $\alpha_{m}$ is infinitely additive on $\sigma(X)$, $m=1,2, \cdots$, then $\left\{\alpha_{m}\right\}_{m=1}^{\infty}$ is weakly convergent if, and only if, $\lim _{m} \alpha_{m}(E)$ exists for every $E$ \& $\sigma(X)$.

In the case where $X$ is a locally compact Hausdorff space, $\sigma(X)$ the algebra generated by the open subsets of $X$, and $\left\{\alpha_{m}\right\}_{m=1}^{\infty}$ is a sequence of Radon measures on $X$, we have, from Theorem 3.4 and Lemma 3.1, the following result of GroTHENDIECK [5].

- The importance of this cannot be overemphasized. Indeed, if this were not the case, then in order to apply Lemma 3.5 we would have to reconstruct $F, T$, and $B^{\prime}(T)$. However, we would have no assurance that $\beta_{m}(E) \rightarrow 0$ for $E$ in the reconstructed $F$. 
Theorem 3.5. Suppose $X, \sigma(X)$, and $\left\{\alpha_{m}\right\}_{m=1}^{\infty}$ satisfy the description given in the preceding paragraph. Then $\left\{\alpha_{m}\right\}_{m=1}^{\infty}$ is a weakly convergent sequence if, and only if, $\lim _{m} \alpha_{m}(E)$ exists for each open set $E \subset X$.

We can apply Theorem 3.4 to some problems in integration. In this direction we have the following results.

Theorem 3.6. If $\sigma(X)$ is a sigma algebra and $\alpha_{m}$ is infinitely additive on $\sigma(X)$, $m=1,2, \cdots$, then $\lim _{m} \int f d \alpha_{m}$ exists for each bounded and measurable function $f$ relative to $\sigma(X)$ if, and only if, $\left\{\alpha_{m}\right\}_{m=1}^{\infty}$ is a weakly convergent sequence.

Theorem 3.7. If $\sigma(X)$ is a sigma algebra and $H_{0}$ a subset of $H(X)$ such that each element of $H_{0}$ is infinitely additive, then a necessary and sufficient condition that each sequence $\left\{\alpha_{m}\right\}_{m=1}^{\infty}$ in $H_{0}$ contain a subsequence $\left\{\beta_{m}\right\}_{m=1}^{\infty}$ such that $\lim _{m} \int f d \beta_{m}$ exists for every bounded and measurable function $f$ relative to $\sigma(X)$ is that $H_{0}$ be weakly sequentially compact.

In the case of the Radon measures, the problem of Theorem 3.6 (existence of $\lim _{m} \int f d \alpha_{m}$ ) was discussed by Dieudonné (cf. [4]), and was later characterized in terms of weak convergence by GRoTHENDIECK [5].

4. The Second Embedding Theorem and Uniform Absolute Continuity. We shall now show that weak convergence in $H(X)$ can be characterized through the concept of uniform absolute continuity. In order to do this we first develop a weak convergence criteria for the general NLC space of bounded functions over any set and where the norm is defined via the least upper bound. This theory, in turn, hinges on the fact that we can embed separable subspaces of the least upper bound spaces into $C[0,1]$ (the continuous functions on $[0,1]$ ) in essentially the same manner that we embedded $H^{\prime}(X)$ into $B[0,1]$ and then interact the results of this embedding with $H^{\prime}(X)$.

With regard to notation, if $X$ is a set, then we use $G(X)$ to denote the NLC space of real-valued and bounded functions on $X$; if $T$ is a linearly ordered set having properties (1) and (2) of Theorem 2.1, then $C(T)$ is the subspace of $G(T)$ of continuous functions. If $f \varepsilon G(X)$, then $\|f\|$ denotes the norm of $f$ as an element of $G(X)$. The distinction in norm notation is important, since in some instances we will discuss a function $f$ which is in both $H(X)$ and $G(\sigma(X))$ and compare the norm $V(f, X)$ with the norm $\|f\|$.

Theorem 4.1. If $G^{\prime}(X)$ is a separable subspace of $G(X)$, then there exists a linearly ordered set $T$ such that $T$ has the properties (1) and (2) of Theorem 2.1 and such that $G^{\prime}(X)$ is isomorphically isometric to a subspace $C^{\prime}(T)$ of $C(T)$, where $C(T)$ denotes the NLC space of continuous functions of $T .^{7}$

Proof. If $E \subset X, \epsilon>0$, and $f \varepsilon G(X)$, then $\mathrm{O}(f, E)<\epsilon$, "oscillation of $f$ on $E$ " means that $\left|f\left(x_{1}\right)-f\left(x_{2}\right)\right|<\epsilon$ for $x_{1}$ and $x_{2}$ in $E$. Let $\left\{f_{m}\right\}_{m=1}^{\infty}$ be a dense

7 This is known (cf. [2], p. 185). However, the constructions in our proof are needed in the remainder of this paper. 
set in $G^{\prime}(X)$. Now the proof of Theorem 4.1 proceeds exactly as the proof of Theorem 2.1; however, in this case the partitions $\left\{E_{i s}\right\}_{i=1}^{n_{s}}, s=1,2, \cdots$, are subsets of $X$ that are selected so that $\mathrm{O}\left(f_{m}, E_{i s}\right)<1 / s$ for $i=1,2, \cdots, n_{s}$ and $m=1,2, \cdots, s$. Again, the elements $t$ of $T$ are defined as nested sequences $\left\{H_{s}\right\}_{s=1}^{\infty}$ where, for each $s, H_{s}=E_{i s}$ for some existing $i \leqq n_{s}$, and once more we shall call the nested sequences $\left\{H_{a}\right\}_{s=1}^{\infty} T$-chains. It is apparent that $T$ satisfies (1) and (2) of Theorem 2.1. In order to construct $C^{\prime}(T)$, let $t \varepsilon T,\left\{H_{p}\right\}_{p=1}^{\infty}$ be the $T$-chain defining $t$, and $x_{p} \varepsilon H_{p}$ for $p=1,2, \ldots$. Since $\mathrm{O}\left(f_{m}, H_{p}\right)<1 / p$ for $m \leqq p$, we see that, for each $m$, the sequence $\left\{f_{m}\left(x_{p}\right)\right\}_{p=1}^{\infty}$ is a Cauchy sequence and, moreover, if $x_{p}^{\prime} \varepsilon H_{p}, p=1,2, \cdots$, then $\lim _{p} f_{m}\left(x_{p}\right)=\lim _{p} f_{m}\left(x_{p}^{\prime}\right)$ for every $m$. Hence, in this manner, we uniquely define for each $m$ and each $t$ the number $h_{m}(t)=\lim _{p} f_{m}\left(x_{p}\right)$ and since $\left|h_{m}(t)\right| \leqq\left\|f_{m}\right\|$, we have $h_{m} \varepsilon G(T)$ and, in fact, $\left\|h_{m}\right\| \leqq\left\|f_{m}\right\|$. Suppose now that there exist an integer $m$ and an $\eta>0$ such that $\left\|f_{m}\right\|=\left\|h_{m}\right\|+\eta$. If $x$ is an element of $X$ such that $\left|\left(\left|f_{m}(x)\right|-\left\|f_{m}\right\|\right)\right|<$ $\frac{1}{2} \eta,\left\{H_{p}\right\}_{p=1}^{\infty}$ is a $T$-chain such that $x \varepsilon H_{p}$ for every $p$ (such a chain obviously exists), and $t$ is the element of $T$ defined by $\left\{H_{p}\right\}_{p=1}^{\infty}$, then $h_{m}(t)=f_{m}(x)$ and

$$
|| f_{m}\left\|-\frac{1}{2} \eta \leqq\left|h_{m}(t)\right| \leqq|| h_{m}\right\| \text {. }
$$

Consequently $\left\|h_{m}\right\|=\left\|f_{m}\right\|$, and the same type of argument easily yields that $G^{\prime}(X)$ and the closed linear manifold generated by $\left\{h_{m}\right\}_{m=1}^{\infty}$, say $C^{\prime}(T)$, in $G(T)$ are isomorphically isometric.

It remains only to show that the elements of $C^{\prime}(T)$ are continuous functions on $T$. Before doing this, let us note that the topology described for the set $T$ of Theorem 2.1 remains valid for the present set. Suppose now that $m$ is an integer and $t_{0} \varepsilon T$. We shall show that $h_{m}$ is continuous at $t_{0}$ from, say, the right. The case where $t_{0}$ is the left member of a gap pair $\left(t^{\prime}, t^{\prime \prime}\right)$ is self-evident, so we shall assume that $\left\{t_{i}\right\}_{i=1}^{\infty}$ is a strictly decreasing sequence approaching $t_{0}$ from the right. Let $\left\{H_{p}\right\}_{p=1}^{\infty}$ be the $T$-chain defining $t_{0}, \epsilon>0$, and $s$ an integer such that $m \leqq s$ and $s^{-1}<\frac{1}{2} \epsilon$. There exists $i_{0}>0$ such that if $i \geqq i_{0}$ and $\left\{H_{p}^{i}\right\}_{p=1}^{\infty}$ is the $T$-chain defining $t_{i}$, then $H_{s}^{i}=H_{s}$. Consequently, if $x \varepsilon H_{s}$ and $i \geqq i_{0}$, then

$$
\left|h_{m}\left(t_{0}\right)-h_{m}\left(t_{i}\right)\right| \leqq\left|h_{m}\left(t_{0}\right)-f_{m}(x)\right|+\left|f_{m}(x)-h_{m}\left(t_{i}\right)\right|<\frac{1}{2} \epsilon+\frac{1}{2} \epsilon=\epsilon,
$$

so that $h_{m}$ is continuous at $t_{0}$ from the right.

This concludes the proof of Theorem 4.1.

As we already remarked, the topology of the set $T$ of Theorem 4.1 has been described in the proof of Theorem 2.1. Consequently, using the homeomorphism $\delta$ between $T$ and $[0,1]$, constructed in the proof of Theorem 2.2, we have the following result of BANACH.

Corollary 4.1.1. The space $G^{\prime}(X)$ is isomorphically isometric to a subspace $C^{\prime}[0,1]$ of $C[0,1]$, where $C[0,1]$ is the $N L C$ space of continuous functions on $[0,1]$.

From Corollary 4.1.1 we get

Corollary 4.1.2. If $k_{m} \varepsilon C^{\prime}(T)$, then $\left\{k_{m}\right\}_{m=1}^{\infty}$ is weakly convergent if, and only 
if, there exists $M>0$ such that $\left\|k_{m}\right\|<M, m=1,2, \cdots$, and $\lim _{m} k_{m}(t)$ exists for every $t \in T$.

We shall now obtain a general weak convergence theorem for $G(X)$. If $f_{m} \varepsilon G(X)$, $m=1,2, \cdots$, then $S\left(\left\{f_{m}\right\}_{m=1}^{\infty}\right)$ denotes the collection of all sequences $\left\{x_{i}\right\}_{i=1}^{\infty}$ such that $x_{i} \varepsilon X, i=1,2, \cdots$, and $\lim _{i} f_{m}\left(x_{i}\right)$ exists for every $m$.

Theorem 4.2. If $f_{m} \varepsilon G(X), m=1,2, \cdots$, then $\left\{f_{m}\right\}_{m=1}^{\infty}$ is weakly convergent if, and only if, the sequence $\left\{\left\|f_{m}\right\|\right\}_{m=1}^{\infty}$ is bounded and

$$
\lim _{m} \lim _{i} f_{m}\left(x_{i}\right)
$$

exists for every sequence $\left\{x_{i}\right\}_{i=1}^{\infty}$ in $S\left(\left\{f_{m}\right\}_{m=1}^{\infty}\right)$.

Proof. For the necessity let us note, since $\left\{x_{i}\right\}_{i=1}^{\infty}$ is in $S\left(\left\{f_{m}\right\}_{m=1}^{\infty}\right)$, that if we define $L\left(f_{m}\right)=\lim f_{m}\left(x_{i}\right)$, then $L$ can be extended to a bounded linear operator over the linear manifold generated by $\left\{f_{m}\right\}_{m=1}^{\infty}$ in $G(X)$. Hence, by the HahnBanach theorem and the definition of weak convergence, (4.2a) is necessary. The boundedness of $\left\{\left\|f_{m}\right\|\right\}_{m=1}^{\infty}$ is, of course, necessary. For the sufficiency, let $G^{\prime}(X)$ denote the closed linear manifold generated by $\left\{f_{m}\right\}_{m=1}^{\infty}, T$ and $C^{\prime}(T)$ the set and space of Theorem 4.1, and $S^{\prime}$ a subset of $S\left(\left\{f_{m}\right\}_{m=1}^{\infty}\right)$ such that $\left\{x_{i}\right\}_{i=1}^{\infty} \varepsilon S^{\prime}$ if, and only if, there exists a $T$-chain $\left\{E_{p}\right\}_{p=1}^{\infty}$ defining an element $t$ of $T$ and an increasing sequence $\left\{n_{i}\right\}_{p=1}^{\infty}$ of positive integers such that $x_{i} \varepsilon H_{n_{i}}$, $i=1,2, \cdots$. Hence if $h_{m}$ is the image of $f_{m}$ in $C^{\prime}(T), m=1,2, \cdots$, then the

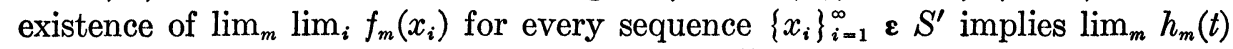
exists for every $t$ in $T$. The boundedness of $\left\{\left\|f_{m}\right\|\right\}_{m=1}^{\infty}$ carries over to $\left\{\left\|h_{m}\right\|\right\}_{m=1}^{\infty}$. Hence, from Corollary 4.1, $\left\{h_{m}\right\}_{m=1}^{\infty}$ is a weakly convergent sequence. This implies $\left\{\left\|f_{m}\right\|\right\}_{m=1}^{\infty}$ is weakly convergent.

This completes the proof of Theorem 4.2. With regard to this theorem, we note that it is not possible to get the boundedness of $\left\{\left\|f_{m}\right\|\right\}_{m=1}^{\infty}$ from (4.2a). For example, let $X$ denote the set of positive integers and for each positive integer $m$ let $f_{m}(x)=m$ or 0 according as $x=m$ or $x \neq m$.

As a consequence of Theorem 4.2, we have

Corollary 4.2.1. If $f_{m} \varepsilon G(X), m=1,2, \cdots$, then $\left\{f_{m}\right\}_{m=1}^{\infty}$ converges weakly to zero if, and only if, $\left\{\left\|f_{m}\right\|\right\}_{m=1}^{\infty}$ is bounded and $\lim _{m} \lim _{i} f_{m}\left(x_{i}\right)=0$ for every sequence $\left\{x_{i}\right\}_{i=1}^{\infty} \varepsilon S\left(\left\{f_{m}\right\}_{m=1}^{\infty}\right)$.

An equivalent way of characterizing when a sequence $\left\{f_{n}\right\}_{n=1}^{\infty}$ in $G(X)$ converges weakly to zero is given in [2], p. 219. In addition to the boundedness of $\left\{\left\|f_{m}\right\|\right\}_{m=1}^{\infty}$, the condition here is that

$$
\lim _{n}\left(\lim _{i} \inf \left|f_{n}\left(x_{i}\right)\right|\right)=0
$$

for every sequence $\left\{x_{i}\right\}_{i=1}^{\infty}$ in $X$. An equivalent formulation of BANACH's condition was given in 1885 by ARzELA, [1], and is called quasi-uniform convergence. Hence it is interesting to note that, in any weakly complete NLC space, weak 
convergence is essentially equivalent to ARzelA's quasi-uniform convergence. ${ }^{8}$

We shall now return to the case of additive functions on $\sigma(X)$. If $\alpha_{m} \varepsilon H(X)$, $m=1,2, \cdots$, and

$$
\alpha_{0}(E)=\sum_{m=1}^{\infty}\left(2^{m}\left[1+V\left(\alpha_{m}, X\right)\right]\right)^{-1} V\left(\alpha_{m}, E\right),
$$

$E \varepsilon \sigma(X)$, then $\alpha_{0} \varepsilon H(X)$ and, for each $m, \alpha_{m}$ is absolutely continuous with respect to $\alpha_{0}$. The sequence $\left\{\alpha_{m}\right\}_{m=1}^{\infty}$ is said to be uniformly absolutely continuous with respect to $\alpha_{0}$ if for each $\epsilon>0$ there exists $\epsilon^{\prime}>0$ such that if $E \varepsilon \sigma(X)$ and $\alpha_{0}(E)<\epsilon^{\prime}$, then $\left|\alpha_{m}(E)\right|<\epsilon$ for $m=1,2, \cdots$.

Theorem 4.3. If $\alpha_{m} \varepsilon H(X), m=1,2, \cdots$, and $\alpha_{0}$ is the function constructed from $\left\{\alpha_{m}\right\}_{m=1}^{\infty}$ in accordance with (4.3a), then $\left\{\alpha_{m}\right\}_{m=1}^{\infty}$ is a weakly convergent sequence if, and only if, $\lim _{m} \alpha_{m}(E)$ exists for each $E$ in $\sigma(X)$ and $\left\{\alpha_{m}\right\}_{m=1}^{\infty}$ is uniformly absolutely continuous with respect to $\alpha_{0}$.

Proof. Sufficiency. Suppose $\left\{E_{p}\right\}_{p \geqq 1} \varepsilon \Gamma(\sigma(X))$. If $\left\{E_{p}\right\}_{p \geqq 1}$ is a finite system, then the pointwise convergence of $\left\{\alpha_{m}\right\}_{m=1}^{\infty}$ implies the validity of (3.1b). If $\left\{E_{p}\right\}_{p \geqq 1}$ is an infinite system, then, because $E_{i} \cdot E_{i}=\theta, i \neq j, \sum_{p=1}^{\infty} \alpha_{0}\left(E_{p}\right)$ is a convergent series. This, together with the pointwise convergence of $\left\{\alpha_{m}\right\}_{m=1}^{\infty}$ and the uniform absolute continuity of $\left\{\alpha_{m}\right\}_{m=1}^{\infty}$ with respect to $\alpha_{0}$, implies the validity of (3.1b). It follows from Corollary 3.1.1 that $\left\{\alpha_{m}\right\}_{m=1}^{\infty}$ is weakly convergent.

Necessity. Obviously the weak convergence of $\left\{\alpha_{m}\right\}_{m=1}^{\infty}$ implies the pointwise convergence of $\left\{\alpha_{m}\right\}_{m=1}^{\infty}$. Suppose now that $\left\{\alpha_{m}\right\}_{m=1}^{\infty}$ is not uniformly absolutely continuous with respect to $\alpha_{0}$. Hence there exist an $\epsilon>0$, a sequence $\left\{\delta_{i}\right\}_{i=1}^{\infty}$ of positive numbers such that $\delta_{i}>\delta_{i+1} \rightarrow 0$, a sequence $\left\{E_{i}\right\}_{i=1}^{\infty}$ of elements of $\sigma(X)$, and an increasing sequence $\left\{n_{i}\right\}_{i=1}^{\infty}$ of positive integers, such that (1) $V\left(\alpha_{0}, E_{i}\right)<\delta_{i}, i=1,2, \cdots$, (2) $V\left(\alpha_{n_{i}}, E_{i}\right)<20 \epsilon, i=1,2, \cdots$, (3) if $s<i$ and $E \varepsilon \sigma(X)$ such that $V\left(\alpha_{0}, E\right)<\delta_{i}$, then $V\left(\alpha_{n_{a}}, E\right)<\epsilon / 2^{s+i}$, and (4) if $i<s$, then $\left|\alpha_{n_{s}}\left(E_{i}\right)\right|<\epsilon / 2^{s+i}$. Now, for each $i$, there exists a subset $E_{i}^{\prime}$ of $E_{i}$ such that $E_{i}^{\prime} \varepsilon \sigma(X)$ and $\left|\alpha_{n i}\left(E_{i}^{\prime}\right)\right|>10 \epsilon$. At this point it may be necessary to select subsequences of $\left\{E_{i}^{\prime}\right\}_{i=1}^{\infty}$ and $\left\{\alpha_{n_{i}}\right\}_{i=1}^{\infty}$ in order to retain (4) above. We shall assume this is done and also, for convenience, take $E_{i}^{\prime}=E_{i}, \alpha_{n_{i}}=i$, and $\alpha_{i}\left(E_{i}\right)>10 \epsilon$.

At this stage of the proof we shall digress and place our problem in a different setting. To this end, we note that the functions $\alpha_{m}, m=1,2, \cdots$, can be considered as elements of the NLC space $G(\sigma(X))$. Also, if $H^{\prime}(X)$ and $G^{\prime}(\sigma(X))$ denote the closed linear subspaces generated by $\left\{\alpha_{m}\right\}_{m=1}^{\infty}$ in $H(X)$ and $G(\sigma(X))$ respectively, then $H^{\prime}(X)$ and $G^{\prime}(\sigma(X))$ are equivalent; i.e. $H^{\prime}(X)$ and $G(\sigma(X))$ are algebraically isomorphic and if $f_{\varepsilon} H^{\prime}(X)$ and $f_{\varepsilon} G^{\prime}(\sigma(X))$, then $\|f\| \leqq$ $V(f, X) \leqq 2\|f\|$. Hence weak convergence is invariant between these spaces so that a sequence $\left\{f_{m}\right\}_{m=1}^{\infty}$ in $H^{\prime}(X)$ is weakly convergent as elements of $H^{\prime}(X)$

\footnotetext{
${ }^{8}$ Cf. our Theorems 4.2 and 5.1 and Lemma 1.1 in [13].
} 
if, and only if, $\left\{f_{m}\right\}_{m=1}^{\infty}$ is a weakly convergent sequence of elements of $G^{\prime}(\sigma(X))$. Using this setting and the functions $\left\{\alpha_{m}\right\}_{m=1}^{\infty}$ and the sets $\left\{E_{i}\right\}_{i=1}^{\infty}$ of the last paragraph, we shall complete our proof of the necessity part of Theorem 4.3 by deriving a contradiction to (4.2a).

We start by setting $K_{1}=H_{1}=E_{1}, \alpha_{1}=\alpha_{m_{1}}, \alpha_{2}=\alpha_{m_{3}}, \alpha_{3}=\alpha_{m_{3}}$, and consider

$$
\alpha_{m_{\mathrm{a}}}\left(E_{1}+E_{3}\right)+\alpha_{m_{\mathrm{a}}}\left(E_{1} \cdot E_{3}\right)=\alpha_{m_{\mathrm{s}}}\left(E_{1}\right)+\alpha_{m_{\mathrm{s}}}\left(E_{3}\right) .
$$

Clearly, one of the numbers $\alpha_{m_{3}}\left(E_{1}+E_{3}\right)$ and $\alpha_{m_{3}}\left(E_{1} \cdot E_{3}\right)$ exceeds $4 \epsilon$. We shall denote by $H_{3}$ that one of the sets $E_{1}+E_{3}$ and $E_{1} \cdot E_{3}$ for which the value of $\alpha_{m_{3}}$ exceeds $4 \epsilon$. If $H_{3}=E_{1} \cdot E_{3}$, then $K_{3}$ denotes $H_{1}-H_{3}$, and if $H_{3}=$ $E_{1}+E_{3}$, then $K_{3}$ denotes $H_{3}$. Now

$$
\alpha_{m_{1}}\left(K_{3}\right)=\alpha_{m_{1}}\left(H_{1}-H_{\mathrm{a}}\right)=\alpha_{m_{1}}\left(H_{1}\right)-\alpha_{m_{1}}\left(H_{3}\right)>10 \epsilon-\frac{1}{4} \epsilon
$$

or

$\alpha_{m_{1}}\left(K_{3}\right)=\alpha_{m_{1}}\left(H_{3}\right)=\alpha_{m_{1}}\left(E_{1}+E_{3}\right)=\alpha_{m_{1}}\left(E_{1}\right)+\alpha_{m_{1}}\left(E_{3}\right)$

$$
-\alpha_{m_{1}}\left(E_{1} \cdot E_{3}\right)>10 \epsilon-2 \epsilon / 4
$$$$
\left|\alpha_{m_{2}}\left(K_{3}\right)\right|=\left|\alpha_{m_{2}}\left(H_{1}\right)-\alpha_{m_{2}}\left(H_{3}\right)\right|<\frac{\epsilon}{2^{3}}+\frac{\epsilon}{2^{5}}
$$

or

$$
\begin{gathered}
\left|\alpha_{m_{2}}\left(K_{3}\right)\right|=\left|\alpha_{m_{2}}\left(E_{1}\right)+\alpha_{m_{2}}\left(E_{3}\right)-\alpha_{m_{3}}\left(E_{1} \cdot E_{3}\right)\right|<\frac{\epsilon}{2^{3}}+\frac{2 \epsilon}{2^{5}} ; \\
\left|\alpha_{m_{3}}\left(K_{3}\right)\right|=\left|\alpha_{m_{3}}\left(H_{1}\right)-\alpha_{m_{3}}\left(H_{3}\right)\right|>10 \epsilon-\frac{\epsilon}{2^{4}}
\end{gathered}
$$

or

$$
\alpha_{m_{3}}\left(K_{3}\right)=\alpha_{m_{3}}\left(E_{1}+E_{3}\right)>4 \epsilon .
$$

Due to the pointwise convergence of $\left\{\alpha_{m}\right\}_{m=1}^{\infty}$, there exists a least integer, say $m_{4}$, such that $\left|\alpha_{m_{0}}\left(K_{i}\right)\right|<\epsilon / 2^{4+i}, i=1$, We denote $m_{4}+1$ by $m_{5}$ and consider

$$
\alpha_{m_{\mathrm{s}}}\left(K_{3}+E_{m_{\mathrm{s}}}\right)+\alpha_{m_{\mathrm{s}}}\left(K_{3} \cdot E_{m_{\mathrm{s}}}\right)=\alpha_{m_{\mathrm{s}}}\left(K_{3}\right)+\alpha_{m_{\mathrm{s}}}\left(E_{m_{\mathrm{s}}}\right) .
$$

Hence one of the numbers $\alpha_{m_{5}}\left(K_{8}+E_{m_{8}}\right)$ and $\alpha_{m_{5}}\left(K_{3} \cdot E_{m_{\mathrm{s}}}\right)$ exceeds $4 \epsilon$, and we shall denote by $H_{5}$ that one of the sets $K_{3}+E_{m_{6}}$ and $K_{3} \cdot E_{m_{8}}$ for which the value of $\alpha_{m_{5}}$ exceeds $4 \epsilon$. If $H_{5}=K_{3} \cdot E_{m_{5}}$, then $K_{5}$ denotes $K_{3}-H_{5}$, and if $H_{5}=K_{3}+E_{m_{5}}$, then $K_{5}$ denotes $H_{5}$. A simple computation gives

$$
\begin{gathered}
\left|\alpha_{m_{1}}\left(K_{5}\right)\right|>10 \epsilon-\frac{\epsilon}{2^{2}}-\frac{\epsilon}{2^{3}},\left|\alpha_{m_{2}}\left(K_{5}\right)\right|<\frac{\epsilon}{2^{3}}+\frac{\epsilon}{2^{5}}+\frac{\epsilon}{2^{7}},\left|\alpha_{m_{0}}\left(K_{5}\right)\right|>4 \epsilon-\frac{\epsilon}{7}, \\
\left|\alpha_{m_{\diamond}}\left(K_{5}\right)\right|<\frac{\epsilon}{2^{5}}+\frac{\epsilon}{2^{7}}, \quad\left|\alpha_{m_{s}}\left(K_{5}\right)\right|>4 \epsilon .
\end{gathered}
$$


Our procedure, though complicated, is evident. We construct a sequence $\left\{K_{2 i-1}\right\}_{i=1}^{\infty}$ of elements of $\sigma(X)$ and select a subsequence $\left\{\alpha_{m_{i}}\right\}_{i=1}^{\infty}$ such that (1) $\lim _{s} \alpha_{m i}\left(K_{2 s-1}\right)$ exists for every $i$, i.e. $\left\{K_{2 s-1}\right\} \varepsilon S\left(\left\{\alpha_{m_{i}}\right\}_{i=1}^{\infty}\right)$, where $\alpha_{m}$, are regarded as elements of $G^{\prime}(\sigma(X))$, (2) $\left|\lim _{i} \lim _{s} \alpha_{m, i}\left(K_{2 s-1}\right)\right|<\epsilon$, and (3) $\mid \lim _{i}$ inf $\left[\lim _{s} \alpha_{m, i-1}\left(K_{2 s-1}\right)\right] \mid>3 \epsilon$. In order to see that (1) is valid, we note that for each $i$ either $K_{2 i-1} \subset K_{2 i+1}$ or $K_{2 i+1} \subset K_{2 i-1}$ and

$$
\left|\alpha_{m_{s}}\left(K_{2 i-1}\right)-\alpha_{m_{s}}\left(K_{2 j+1}\right)\right|<\frac{\epsilon}{2^{s-1}} \sum_{p=i}^{j-1} \frac{1}{2^{p-1}}
$$

for $s<i<j$, so that $\left\{\alpha_{m},\left(K_{2 i-1}\right)\right\}_{i=1}^{\infty}$ is a Cauchy sequence. Hence, in view of (1), we see that (2) and (3) contradict (4.2a) of Theorem 4.2.

This completes the proof of Theorem 4.3.

5. Concluding Remarks. It is possible to interpret some of our results for the case of a general NLC space. If $R$ is such a space, then $R$ can be isomorphically and isometrically embedded into $R^{* *}$ (via the natural embedding); hence we can regard $R$ as a proper subspace of $G\left(R_{1}{ }^{*}\right)$, where $R_{1}{ }^{*}$ is the unit sphere in $R^{*}$. Hence Theorem 4.2 gives

Theorem 5.1. If $R$ is NLC and $x_{m} \varepsilon R, m=1,2, \cdots$, then $\left\{x_{m}\right\}_{m=1}^{\infty}$ is a weakly convergent sequence if, and only if, $\left\{\left\|x_{m}\right\|\right\}_{m=1}^{\infty}$ is a bounded sequence and $\lim _{m} \lim _{i} L_{i}\left(x_{m}\right)$ exists for every sequence $\left\{L_{i}\right\}_{i=1}^{\infty} \varepsilon R_{1}^{*}$ such that $\lim _{2} L_{i}\left(x_{m}\right)$ exists for every $m$.

This theorem can be derived directly by other means. The sufficiency, of course, is trivial $\left(L_{i}=L\right)$, and the necessity follows from the existence of BANACH's generalized limit.

In order to distinguish between the applicability of Theorems 4.2 and 5.1, we shall apply Theorem 4.2 to the space $(m)$ of bounded sequences. In the case of the space $(m)$, Theorem 4.2 readily yields

Theorem 5.2. If $X_{n}=\left\{x_{n_{i}}\right\}_{i=1}^{\infty}$ and $X_{n} \varepsilon(m), n=1,2, \cdots$, then $\left\{X_{n}\right\}_{n=1}^{\infty}$ is a weakly convergent sequence if, and only if, $\left\{\left\|X_{n}\right\|\right\}_{n=1}^{\infty}$ is a bounded sequence and $\lim _{n} \lim _{i} x_{n m i}$ exists for every sequence $\left\{m_{i}\right\}_{n=1}^{\infty}$ of integers such that $\lim _{i} x_{n m}$, exists, $n=1,2, \cdots$.

We note that the above condition requires that $\lim _{n} x_{n i}$ exist for $i=1,2, \cdots$. In the case of weak convergence to zero, the above condition is equivalent to $\lim _{n} \lim _{i}$ inf $\left|x_{n i}\right|=0$ and pointwise convergence (cf. [2], Th. 10, p. 225).

\section{BibliograPhy}

[1] C. Arzela, Sulle funzioni di linee, Memoirie dell'Academia di Bologna, 5 (1885), pp. 225-244.

[2] S. Banach, Theorie des operations lineaires, Warsaw, 1932.

[3] S. Banach \& S. Mazur, Zur Theorie der linearen Dimension, Studia Math., 4 (1933), pp. 100-112. 
[4] J. Dieudonne, Sur la convergences des suites de measures de Radon, Anais da Acad. Brasil. de Ciencias, 23 (1951), pp. 21-38.

[5] A. Grothendieck, Sur les applications linéaires faiblement compactes d'espaces du type $C(K)$, Can. Jour. of Math., 5 (1953), pp. 129-173.

[6] T. H. Hildebrandt, Integration in abstract spaces, Bull. Amer. Nath. Soc., 59 (1953), pp. 111-139.

[7] T. H. Hildebrandt, On bounded linear functional operations, Trans. Amer. Math. Soc., 36 (1934), pp. 868-875.

[8] T. H. Hildebrand, Linear operations on functions of bounded variation, Bull. Amer. Math. Soc., 44 (1938), p. 75.

[9] T. H. HildDebrand, On a theorem in the space $l_{1}$ of absolutely convergent sequences with applications to completely additive set functions, Math. Research Center Report \#62, Madison, Wis., 1958.

[10] S. Kakutani, Concrete representations of abstract $(L)$-spaces and the mean ergodic theorem, Ann. of Math., 42 (1941), pp. 523-537.

[10'] O. Nikodym, Sur les suites convergentes des fonctions parfaitement additive d'ensemble abstrait, Monatsh. Math. u. Phys., 40 (1933), pp. 427-432.

[11] P. Porcelli, On weak convergence in the space of functions of bounded variation, Math. Research Center Report \#39, Madison, Wis., 1958.

[12] P. Porcelli, On weak convergence in the space of functions of bounded variation II, Math. Research Center Report \#68, Madison, Wis., 1958.

[13] S. SirvinT, Weak compactness in Banach sppaces, Studia Math., 11 (1950), pp. 71-94.

Mathematics Research Center, U. S. Army

Madison, Wisconsin 